IJMMS 29:9 (2002) 537-544

PII. S0161171202007809

http://ijmms.hindawi.com

(C) Hindawi Publishing Corp.

\title{
GLOBAL ATTRACTIVITY IN A GENOTYPE SELECTION MODEL
}

\section{XIAOPING LI}

Received 20 July 2001

We obtain a sufficient condition for the global attractivity of the genotype selection model $y_{n+1}=y_{n} e^{\beta_{n}\left(1-2 y_{n-k}\right)} /\left(1-y_{n}+y_{n} e^{\beta_{n}\left(1-2 y_{n-k}\right)}\right), n \in \mathbb{N}$. Our results improve the results established by Grove et al. (1994) and Kocić and Ladas (1993).

2000 Mathematics Subject Classification: 39A10, 39A12.

1. Introduction. Let $\mathbb{Z}$ denote the set of all integers. For $a, b \in \mathbb{Z}$, define $\mathbb{N}(a)=$ $\{a, a+1, \ldots\}, \mathbb{N}=\mathbb{N}(0)$, and $\mathbb{N}(a, b)=\{a, a+1, \ldots, b\}$ when $a \leq b$.

Consider the following nonlinear delay difference equation:

$$
y_{n+1}=\frac{y_{n} e^{\beta_{n}\left(1-2 y_{n-k}\right)}}{1-y_{n}+y_{n} e^{\beta_{n}\left(1-2 y_{n-k}\right)}}, \quad n \in \mathbb{N},
$$

where $k \in \mathbb{N}$ and $\left\{\beta_{n}\right\}$ is a sequence of positive real numbers.

When $k=0$ and $\beta_{n} \equiv \beta$ for all $n \in \mathbb{N}$, (1.1) was introduced by May [2, pages 513-560] as an example of a map generated by a simple model for frequency-dependent natural selection. The local stability of the equilibrium $\bar{y}=1 / 2$ of (1.1) was investigated by May [2]. In [1] (see also [3]), Grove further investigated the stability of the equilibrium $\bar{y}=1 / 2$ of (1.1) and proved that when $\beta_{n} \equiv \beta$, the equilibrium $\bar{y}=1 / 2$ of (1.1) is locally asymptotically stable if $0<\beta<4 \cos (k \pi /(2 k+1))$ and is unstable if $0<\beta<$ $4 \cos (k \pi /(2 k+1))$. Furthermore, if

$$
0<\beta \leq \frac{2}{k}, \quad k \in \mathbb{N}(1)
$$

Then this equilibrium is a global attractor of all solution $\left\{y_{n}\right\}$ of (1.1) with initial conditions $y_{-k}, y_{-k+1}, \ldots, y_{0} \in(0,1)$.

On the basis of computer observations, the authors of [1] also observe that condition (1.2) is probably far from sharp when $k \in \mathbb{N}(2)$. Therefore, it is highly desirable to improve condition (1.2).

The purpose of this paper is to obtain new sufficient conditions for the global attractivity of the equilibrium $\bar{y}=1 / 2$ of (1.1). Our main result is the following theorem.

THEOREM 1.1. Assume that $\left\{\beta_{n}\right\}$ is a positive sequence which satisfies

$$
\sum_{i=n-k}^{n} \beta_{i} \leq 3+\frac{1}{k+1},
$$


for all large $n$, and

$$
\sum_{i=0}^{\infty} \beta_{i}=\infty .
$$

Then every solution $\left\{y_{n}\right\}$ of (1.1) with initial conditions $y_{-k}, y_{-k+1}, \ldots, y_{0} \in(0,1)$ will tend to $\bar{y}=1 / 2$.

COROLlary 1.2. Assume that $\beta_{n} \equiv \beta$ for all $n \in \mathbb{N}$ and

$$
\beta \leq \frac{3}{k+1}+\frac{1}{(k+1)^{2}} .
$$

Then every solution $\left\{y_{n}\right\}$ of (1.1) with initial conditions $y_{-k}, y_{-k+1}, \ldots, y_{0} \in(0,1)$ will tend to $\bar{y}=1 / 2$.

It is easy to see that when $k \in \mathbb{N}(2),(1.5)$ is an improvement on (1.2).

By a solution of (1.1), we mean a sequence $\left\{y_{n}\right\}$ that is defined for $n \in \mathbb{N}(-k)$ and that satisfies (1.1) for $n \in \mathbb{N}$. If $a_{-k}, a_{-k+1}, \ldots, a_{0}$ are $k+1$ given constants, then (1.1) has a unique solution satisfying the initial conditions

$$
x_{i}=a_{i} \quad \text { for } i \in \mathbb{N}(-k, 0) .
$$

For the sake of convenience, throughout, we use the convention

$$
\sum_{n=i}^{j} r_{n} \equiv 0, \quad \text { whenever } j \leq i-1 \text {. }
$$

2. Proof of Theorem 1.1. Let $\left\{y_{n}\right\}$ be a solution of (1.1) with initial conditions $y_{-k}$, $y_{-k+1}, \ldots, y_{0} \in(0,1)$. Then clearly, $y_{n} \in(0,1)$ for all $n \in \mathbb{N}(-k)$. By introducing the substitution

$$
x_{n}=\ln \frac{y_{n}}{1-y_{n}}, \quad n \in \mathbb{N}(-k),
$$

we obtain

$$
\begin{gathered}
\Delta x_{n}+r_{n} f\left(x_{n-k}\right)=0, \quad n \in \mathbb{N}, \\
x_{-k}, x_{-k+1}, \ldots, x_{0} \in(-\infty, \infty),
\end{gathered}
$$

where

$$
\Delta x_{n}=x_{n+1}-x_{n}, \quad r_{n}=\frac{1}{2} \beta_{n}, \quad f(x)=2-\frac{4}{e^{x}+1} .
$$

It is easy to see that

$$
\begin{gathered}
f(0)=0, \quad x f(x)>0 \quad \forall x \in \mathbb{R}, \\
f^{\prime}(x)=\frac{4 e^{x}}{\left(e^{x}+1\right)^{2}} \quad \forall x \in \mathbb{R} .
\end{gathered}
$$


Thus, $f$ is increasing, we also have

$$
f^{\prime}(x)<\frac{4 e^{x}}{\left(2 \sqrt{e^{x}}\right)^{2}}=1 \text { for } x \neq 0,
$$

which implies that

$$
|f(x)|<|x| \text { for } x \neq 0 \text {. }
$$

Define $h$ as follows

$$
h(x)=\max \{f(x),-f(-x)\} \quad \text { for } x>0 .
$$

We have from (2.5), (2.8), and the increasing property of $f$ that $h(x)$ is increasing in $[0, \infty)$, and

$$
|f(x)| \leq h(|x|)<|x| \text { for } x \neq 0 .
$$

We will now prove that

$$
\lim _{n \rightarrow \infty} x_{n}=0
$$

There are two cases to consider.

CASE 1. The sequence $\left\{x_{n}\right\}$ is eventually nonnegative or eventually nonpositive. We assume that $\left\{x_{n}\right\}$ is eventually nonnegative, then there exists an integer $n_{0} \in \mathbb{N}(k)$ such that $x_{n-k} \geq 0$ for all $n \in \mathbb{N}\left(n_{0}\right)$. By (2.2), we have $\Delta x_{n} \leq 0$ for all $n \in \mathbb{N}\left(n_{0}\right)$ and there exists $a \geq 0$ such that

$$
\lim _{n \rightarrow \infty} x_{n}=a .
$$

If $a>0$, by the increasing property of $f$, it follows that

$$
\Delta x_{n} \leq-r_{n} f(a) \quad \forall n \in \mathbb{N}\left(n_{0}+k\right) .
$$

Summing (2.13) from $n_{0}+k$ to $n-1$ and using (1.4), we have

$$
x_{n}-x_{n_{0}+k} \leq-f(a) \sum_{i=n_{0}+k}^{n-1} r_{i} \longrightarrow-\infty \text { as } n \longrightarrow \infty,
$$

which contradicts (2.12). The case when $\left\{x_{n}\right\}$ is eventually nonpositive can be dealt with similarly.

CASE 2. The sequence $\left\{x_{n}\right\}$ is oscillatory. By (1.3) and (2.4), then there exists an integer $n^{*} \in \mathbb{N}(2 k)$ such that

$$
\begin{gathered}
\sum_{i=n-k}^{n} r_{i} \leq \alpha=\frac{3}{2}+\frac{1}{2(k+1)}, \quad n \in \mathbb{N}\left(n^{*}-2 k\right), \\
x_{n^{*}-1} x_{n^{*}} \leq 0, \quad x_{n^{*}} \neq 0 .
\end{gathered}
$$

By virtue of the choice of $n^{*}$, there exists a real number $\lambda \in[0,1)$ such that

$$
x_{n^{*-1}}+\lambda\left(x_{n *}-x_{n^{*-1}}\right)=0 .
$$


Let $l$ be a positive constant such that

$$
\max _{n \in \mathbb{N}\left(n^{*}-2 k-1, n^{*}-1\right)}\left|x_{n}\right| \leq l .
$$

By (2.2), (2.10), (2.18), and the increasing property of $h$, we have

$$
\left|\Delta x_{n}\right| \leq r_{n} h(l), \quad n \in \mathbb{N}\left(n^{*}-1, n^{*}+k-1\right)
$$

Which, together with (2.17), implies that

$$
\begin{aligned}
\left|x_{n-k}\right| & =\left|x_{n-k}-x_{n^{*}-1}-\lambda\left(x_{n^{*}}-x_{n^{*}-1}\right)\right| \\
& =\left|-\sum_{j=n-k}^{n^{*}-2} \Delta x_{j}-\lambda \Delta x_{n^{*}-1}\right| \\
& \leq h(l)\left(\sum_{j=n-k}^{n^{*}-2} r_{j}+\lambda r_{n^{*}-1}\right), \quad n \in \mathbb{N}\left(n^{*}-1, n^{*}+k-1\right) .
\end{aligned}
$$

In view of (2.2), (2.10), and (2.20), we obtain

$$
\left|\Delta x_{n}\right| \leq r_{n} h(l)\left(\sum_{j=n-k}^{n^{*}-2} r_{j}+\lambda r_{n^{*}-1}\right), \quad n \in \mathbb{N}\left(n^{*}-1, n^{*}+k-1\right) .
$$

Now we show that

$$
\left|x_{n}\right| \leq h(l) \quad \forall n \in \mathbb{N}\left(n^{*}, n^{*}+k\right) .
$$

There are two possible cases to consider.

CASE 1. Suppose that $d=\sum_{i=n^{*}}^{n^{*}+k-1} r_{i}+(1-\lambda) r_{n^{*}-1} \leq 1$. By (2.15), (2.17), and (2.21) we have for $n \in \mathbb{N}\left(n^{*}, n^{*}+k\right)$

$$
\begin{aligned}
\left|x_{n}\right|= & \left|x_{n}-x_{n^{*}-1}-\lambda\left(x_{n^{*}}-x_{n^{*}-1}\right)\right| \\
= & \left|\sum_{i=n^{*}}^{n-1} \Delta x_{i}+(1-\lambda) \Delta x_{n^{*}-1}\right| \\
\leq & \sum_{i=n^{*}}^{n^{*}+k-1} r_{i} h(l)\left(\sum_{j=i-k}^{n^{*}-2} r_{j}+\lambda r_{n^{*}-1}\right)+(1-\lambda) r_{n^{*}-1} h(l)\left(\sum_{j=n^{*}-k-1}^{n^{*}-2} r_{j}+\lambda r_{n^{*}-1}\right) \\
= & h(l) \sum_{i=n^{*}}^{n^{*}+k-1} r_{i}\left[\sum_{j=i-k}^{i} r_{j}-\sum_{j=n^{*}}^{i} r_{j}-(1-\lambda) r_{n^{*}-1}\right] \\
& +h(l)(1-\lambda) r_{n^{*}-1}\left[\sum_{j=n^{*}-k-1}^{n^{*}-1} r_{j}-(1-\lambda) r_{n^{*}-1}\right]
\end{aligned}
$$




$$
\begin{aligned}
& \leq h(l)\left[\alpha d-\sum_{i=n^{*}}^{n^{*}+k-1} r_{i} \sum_{j=n^{*}}^{i} r_{j}-(1-\lambda) r_{n^{*}-1} d\right] \\
& =h(l)\left[\alpha d-\frac{1}{2}\left(\sum_{i=n^{*}}^{n^{*}+k-1} r_{i}\right)^{2}-\frac{1}{2} \sum_{i=n^{*}}^{n^{*}+k-1} r_{i}^{2}-(1-\lambda) r_{n^{*}-1} d\right] \\
& =h(l)\left[\alpha d-\frac{1}{2} d^{2}-\frac{1}{2}\left(\sum_{i=n^{*}}^{n^{*}+k-1} r_{i}^{2}+(1-\lambda)^{2} r_{n^{*}-1}^{2}\right)\right] .
\end{aligned}
$$

Since

$$
\sum_{i=n^{*}}^{n^{*}+k-1} r_{i}^{2}+(1-\lambda)^{2} r_{n^{*}-1}^{2} \geq \frac{1}{k+1}\left(\sum_{i=n^{*}}^{n^{*}+k-1} r_{i}+(1-\lambda) r_{n^{*}-1}\right)^{2}=\frac{d^{2}}{k+1}
$$

We obtain

$$
\begin{aligned}
\left|x_{n}\right| & \leq h(l)\left[\alpha d-\left(\frac{1}{2}+\frac{1}{2(k+1)}\right) d^{2}\right] \\
& \leq h(l)\left[\alpha-\left(\frac{1}{2}+\frac{1}{2(k+1)}\right)\right] \\
& =h(l) .
\end{aligned}
$$

CASE 2. Suppose that $d=\sum_{i=n^{*}}^{n^{*}+k} r_{i}+(1-\lambda) r_{n^{*}-1}>1$. In this case, there exists an integer $m \in \mathbb{N}\left(n^{*}, n^{*}+k\right)$ such that

$$
\sum_{i=m}^{n^{*}+k-1} r_{i} \leq 1, \quad \sum_{i=m-1}^{n^{*}+k-1} r_{i}>1
$$

Therefore, there is an $\eta \in(0,1]$ such that

$$
\sum_{i=m}^{n^{*}+k-1} r_{i}+(1-\eta) r_{m-1}=1
$$

By (2.15), (2.17), (2.19), and (2.21), we have for $n \in \mathbb{N}\left(n^{*}, n^{*}+k\right)$

$$
\begin{aligned}
\left|x_{n}\right| & =\left|x_{n}-x_{n^{*}-1}-\lambda \Delta x_{n^{*}-1}\right| \\
& =\left|\sum_{i=n^{*}}^{n-1} \Delta x_{i}+(1-\lambda) \Delta x_{n^{*}-1}\right| \\
& =\sum_{j=n^{*}}^{n^{*}+k-1}\left|\Delta x_{j}\right|+(1-\lambda)\left|\Delta x_{n^{*-1}}\right|
\end{aligned}
$$




$$
\begin{aligned}
& =(1-\lambda)\left|\Delta x_{n^{*}-1}\right|+\sum_{j=n^{*}}^{m-2}\left|\Delta x_{j}\right|+\eta\left|\Delta x_{m-1}\right|+(1-\eta)\left|\Delta x_{m-1}\right|+\sum_{j=m}^{n^{*}+k-1}\left|\Delta x_{j}\right| \\
& \leq h(l)\left((1-\lambda) r_{n^{*}-1}+\sum_{j=n^{*}}^{m-2} r_{j}+\eta r_{m-1}\right)+h(l)(1-\eta) r_{m-1}\left(\sum_{j=m-1-k}^{n^{*}-2} r_{j}+\lambda r_{n^{*}-1}\right) \\
& +h(l) \sum_{j=m}^{n^{*}+k-1} r_{j}\left(\sum_{i=j-k}^{n^{*}-2} r_{i}+\lambda r_{n^{*}-1}\right) \\
& =h(l)\left[(1-\lambda) r_{n^{*}-1}+\sum_{j=n^{*}}^{m-1} r_{j}-(1-\eta) r_{m-1}\right] \\
& +h(l)(1-\eta) r_{m-1}\left[\sum_{j=m-1-k}^{m-1} r_{j}-\sum_{j=n^{*}}^{m-1} r_{j}-(1-\lambda) r_{n^{*}-1}\right] \\
& +h(l) \sum_{j=m}^{n^{*}+k-1} r_{j}\left[\sum_{i=j-k}^{j} r_{i}-\sum_{i=m}^{j} r_{i}-\sum_{i=n^{*}}^{m-1} r_{i}-(1-\lambda) r_{n^{*}-1}\right] \\
& \leq h(l)\left[\alpha-(1-\eta) r_{m-1}-\sum_{j=m}^{n^{*}+k-1} r_{j} \sum_{i=m}^{j} r_{i}\right] \\
& =h(l)\left[\alpha-(1-\eta) r_{m-1}-\frac{1}{2}\left(\sum_{j=m}^{n^{*}+k-1} r_{j}\right)^{2}-\frac{1}{2} \sum_{j=m}^{n^{*}+k-1} r_{j}^{2}\right] \\
& =h(l)\left[\alpha-(1-\eta) r_{m-1}-\frac{1}{2}\left(1-(1-\eta) r_{m-1}\right)^{2}-\frac{1}{2} \sum_{j=m}^{n^{*}+k-1} r_{j}^{2}\right] \\
& =h(l)\left[\alpha-\frac{1}{2}-\frac{1}{2}\left(\sum_{j=m}^{n^{*}+k-1} r_{j}^{2}+(1-\eta)^{2} r_{m-1}^{2}\right)\right] \text {. }
\end{aligned}
$$

Since

$$
\sum_{j=m}^{n^{*}+k-1} r_{j}^{2}+(1-\eta)^{2} r_{m-1}^{2} \geq \frac{1}{n^{*}-m+k+1}\left(\sum_{j=m}^{n^{*}+k-1} r_{j}+(1-\eta) r_{m-1}\right)^{2} \geq \frac{1}{k+1}
$$

We obtain

$$
\left|x_{n}\right| \leq h(l)\left(\alpha-\frac{1}{2}-\frac{1}{2(k+1)}\right)=h(l) .
$$

Furthermore, we can prove that

$$
\left|x_{n}\right| \leq h(l) \quad \forall n \in \mathbb{N}\left(n^{*}\right) .
$$


Assume, for the sake of contradiction, that (2.31) is not true. Then there exists $m_{1} \in$ $\mathbb{N}\left(n^{*}+k+1\right)$ such that $\left|x_{m_{1}}\right|>h(l)$ and $\left|x_{n}\right| \leq h(l)$ for $n \in \mathbb{N}\left(n^{*}, m_{1}-1\right)$. Set

$$
m_{2}=\max \left\{n \in \mathbb{N}\left(n^{*}, m_{1}\right): x_{n-1} x_{n} \leq 0, x_{n} \neq 0\right\} .
$$

In case $m_{1} \leq m_{2}+k$. From (2.10), we have

$$
\max _{n \in \mathbb{N}\left(m_{2}-2 k-1, m_{2}-1\right)}\left|x_{n}\right| \leq h(l)<l .
$$

By a similar method to the proof of (2.22), we obtain

$$
\left|x_{n}\right| \leq h(l) \quad \forall n \in \mathbb{N}\left(m_{2}, m_{2}+k\right)
$$

which contradicts the definition of $m_{1}$. In case $m_{1}-1 \geq m_{2}+k$, it follows from the choice of $m_{1}$ and $m_{2}$ that

$$
x_{n}>0 \quad \text { or } \quad x_{n}<0 \quad \forall n \in \mathbb{N}\left(m_{2}, m_{1}\right) \text {. }
$$

Assume that $x_{n}>0$ for all $n \in \mathbb{N}\left(m_{2}, m_{1}\right)$. (In case $x_{n}<0$, the proof is similar.) From (2.2) we have

$$
\Delta x_{n} \leq 0 \text { for } n \in \mathbb{N}\left(m_{1}-1, m_{1}+k\right)
$$

which implies that

$$
x_{m_{1}} \leq x_{m_{1}-1} \leq h(l) \text {. }
$$

This contradicts the definition of $m_{1}$. Thus (2.31) holds.

From the argument above, we can establish a sequence $\left\{n_{i}\right\}$ of positive integers with $n_{1}=n^{*}, n_{i+1}-n_{i}>2 k$ such that

$$
x_{n_{i}-1} x_{n_{i}} \leq 0, \quad x_{n_{i}} \neq 0,
$$

and a sequence $\left\{z_{i}\right\}$ with $z_{1}=l, z_{i+1}=h\left(z_{i}\right)$ such that

$$
\max _{n \in \mathbb{N}\left(n_{i}-2 k-1, n_{i}-1\right)}\left|x_{n}\right| \leq z_{i}, \quad\left|x_{n}\right| \leq z_{i+1} \quad \forall n \in \mathbb{N}\left(n_{i}\right) .
$$

By (2.10), we obtain

$$
\lim _{i \rightarrow \infty} z_{i}=0
$$

which, together with (2.39), implies that $\lim _{n \rightarrow \infty} x_{n}=0$. The proof is complete.

ACKNOWLEDGMENT. This work was supported by the Science Foundation of Hunan Educational Committee of China. 


\section{REFERENCES}

[1] E. A. Grove, V. L. Kocić, G. Ladas, and R. Levins, Oscillation and stability in a simple genotype selection model, Quart. Appl. Math. 52 (1994), no. 3, 499-508.

[2] G. Iooss, R. H. G. Helleman, and R. Stora (eds.), Chaotic Behavior of Deterministic Systems, North-Holland Publishing, Amsterdam, 1983.

[3] V. L. Kocić and G. Ladas, Global Behavior of Nonlinear Difference Equations of Higher Order with Applications, Mathematics and Its Applications, vol. 256, Kluwer Academic Publishers Group, Dordrecht, 1993.

Xiaoping li: Department of Mathematics, Loudi Teacher's College loudi, Hunan 417000, CHINA

E-mail address: 1d1xpi i@mai 1.1d.hn.cn 


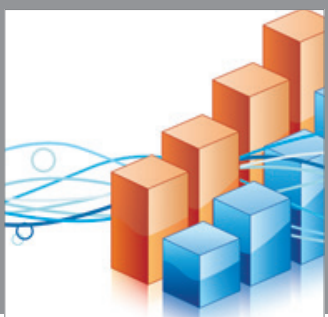

Advances in

Operations Research

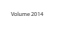

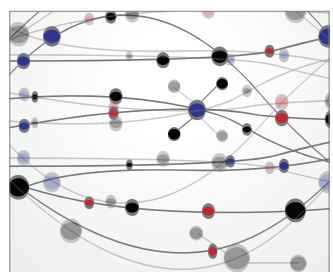

\section{The Scientific} World Journal
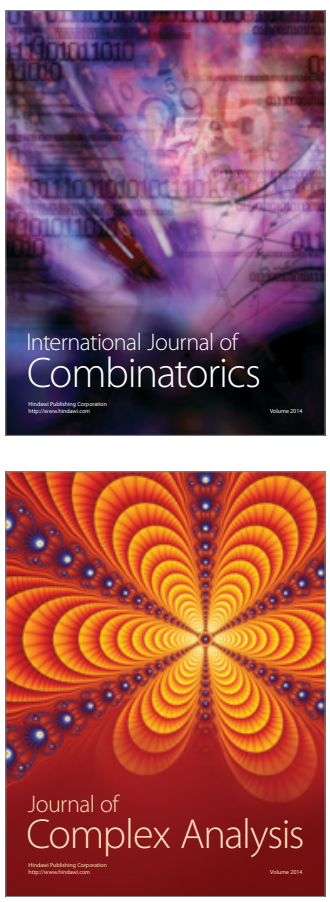

International Journal of

Mathematics and

Mathematical

Sciences
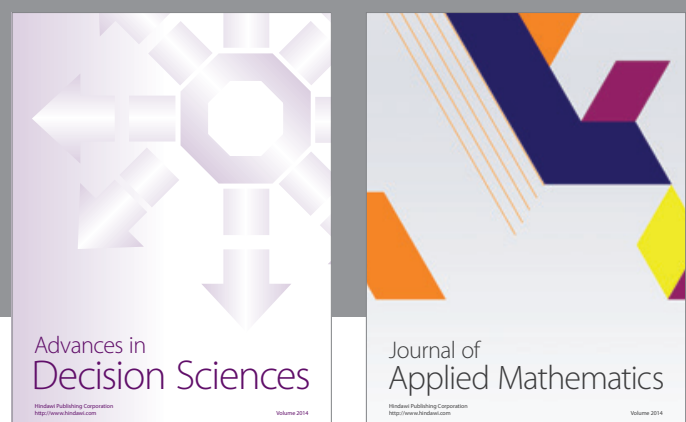

Journal of

Applied Mathematics
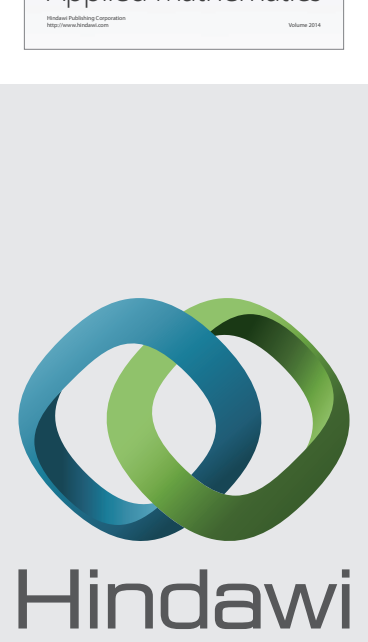

Submit your manuscripts at http://www.hindawi.com
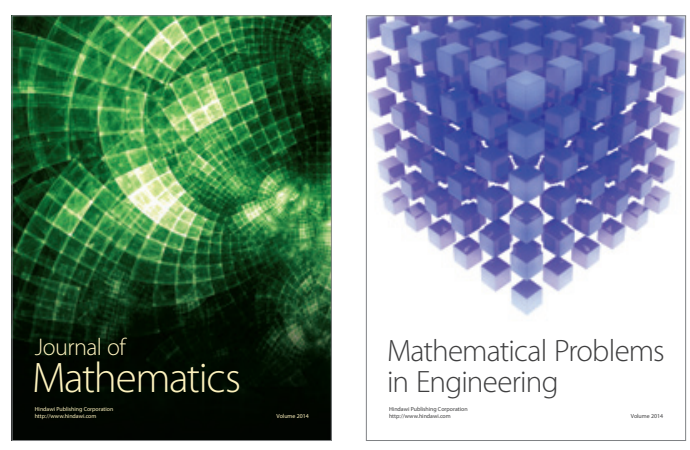

Mathematical Problems in Engineering
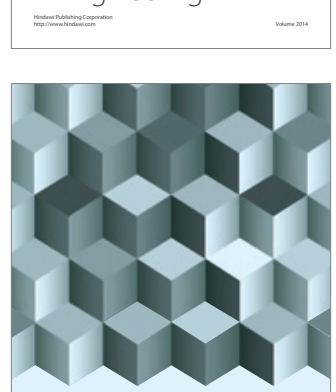

Journal of

Function Spaces
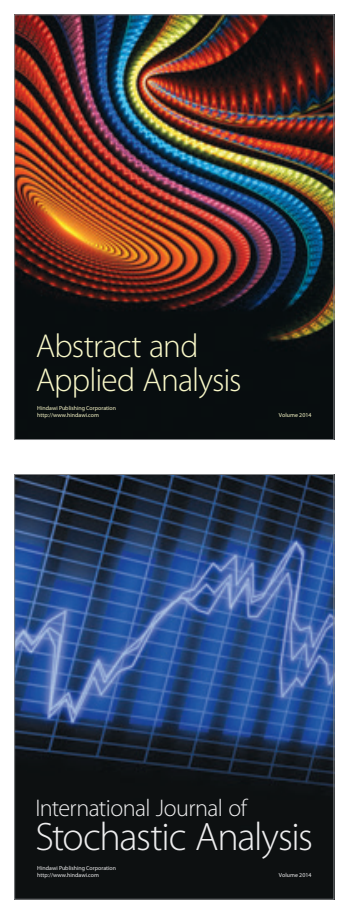

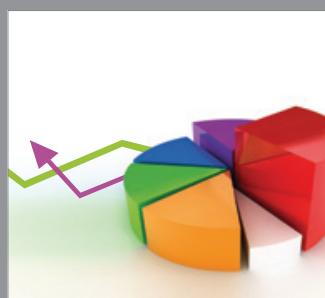

ournal of

Probability and Statistics

Promensencen
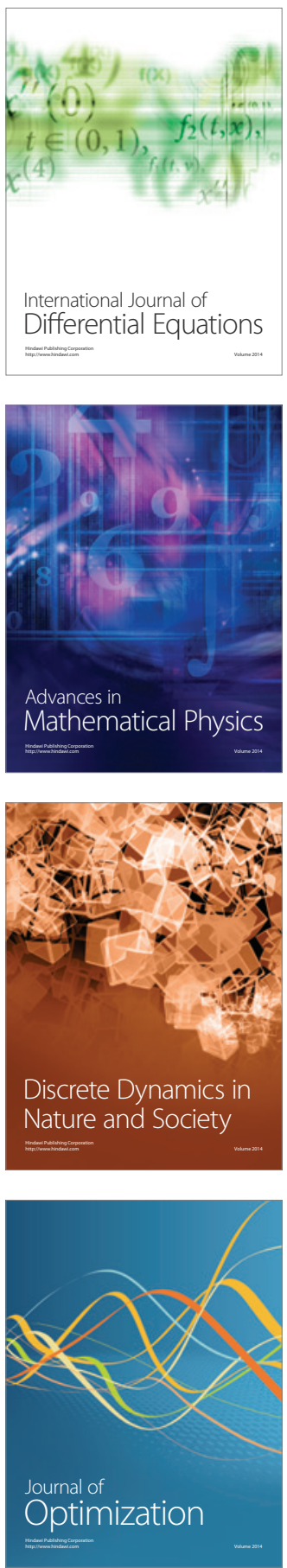